\title{
On Compact Routing for the Internet
}

\author{
Dmitri Krioukov \\ CAIDA \\ dima@caida.org \\ Kevin Fall \\ Intel Research Berkeley \\ kfall@intel.com
}

\author{
kc claffy \\ CAIDA \\ kc@caida.org \\ Arthur Brady \\ Tufts University \\ abrady@cs.tufts.edu
}

\begin{abstract}
The Internet's routing system is facing stresses due to its poor fundamental scaling properties. Compact routing is a research field that studies fundamental limits of routing scalability and designs algorithms that try to meet these limits. In particular, compact routing research shows that shortest-path routing, forming a core of traditional routing algorithms, cannot guarantee routing table (RT) sizes that on all network topologies grow slower than linearly as functions of the network size. However, there are plenty of compact routing schemes that relax the shortest-path requirement and allow for improved, sublinear RT size scaling that is mathematically provable for all static network topologies. In particular, there exist compact routing schemes designed for grids, trees, and Internet-like topologies that offer RT sizes that scale logarithmically with the network size.

In this paper, we demonstrate that in view of recent results in compact routing research, such logarithmic scaling on Internet-like topologies is fundamentally impossible in the presence of topology dynamics or topology-independent (flat) addressing. We use analytic arguments to show that the number of routing control messages per topology change cannot scale better than linearly on Internet-like topologies. We also employ simulations to confirm that logarithmic RT size scaling gets broken by topology-independent addressing, a cornerstone of popular locator-identifier split proposals aiming at improving routing scaling in the presence of network topology dynamics or host mobility. These pessimistic findings lead us to the conclusion that a fundamental re-examination of assumptions behind routing models and abstractions is needed in order to find a routing architecture that would be able to scale "indefinitely."
\end{abstract}

\section{Categories and Subject Descriptors}

C.2.2 [Network Protocols]: Routing protocols;

G.2.2 [Graph Theory]: Graph algorithms, Network problems;

C.2.1 [Network Architecture and Design]: Network topology

\section{General Terms}

Algorithms, Design, Theory

\section{Keywords}

Compact routing, Internet routing, routing scalability

\section{INTRODUCTION}

Despite prevailing concerns among network operators and developers that the current Internet interdomain routing system will not scale to meet the needs of the 21 st century global Internet, networking research has not yet produced a new routing architecture with satisfactory flexibility and mathematically provable scalability characteristics. We believe that we will soon be faced with a critical architectural inflection point posing a significant challenge to scaling the size of the future Internet. The last critical point was reached when the Internet's routing system adopted strong address aggregation using CIDR to handle address scaling in the mid 90's. While CIDR was an extremely effective tactic, most experts agree that the growth behavior of the routing table in the last decade confirms that the type of address management required by CIDR will not suffice to meet future Internet routing needs, and that a fundamental top-to-bottom reexamination of the routing architecture may be required [1].

In this paper we set to work on such a reexamination 1 As we proceed, we first identify, in Section 2, the fundamental causes of Internet routing scalability problems, and how they imply the need for dramatically more efficient routing algorithms with rigorously proven worst-case performance guarantees. Such algorithms exist-they are known collectively as compact routing schemes. They do not and, generally, can not guarantee routing along shortest paths-they stretch them. In Section 3 we juxtapose the formal notion of stretch (or hop stretch) with better-known forms of path inflation present in conventional networking. There is a fundamental trade-off between stretch and routing table (RT) size, and we show in Section 4 why hierarchical routing and addressing fall short in finding an optimal balance point of this trade-off for topologies of interest. We also outline, in the same section, known alternative addressing techniques that compact routing schemes use. We analyze the key ideas behind these schemes in Section 5 . We focus on universal schemes in this section and shift our attention to the schemes specialized for Internet-like topologies in Section 6] All these schemes are static. They ignore any communication overhead their implementations might require. We use analytic arguments in Section 7 to directly prove that recent results in compact routing research render

\footnotetext{
${ }^{1}$ We clarify up front that scalability is only one of several problems of the current Internet routing architecture. Other problems include security, isolation, configuration control, etc. See 2] for a long list of future routing architecture requirements.
} 
fundamentally unscalable the communication costs of any routing algorithm applied to Internet-like topologies. Despite these findings, "locator-identifier split" (LIS) proposals are becoming popular these days, as they are widely believed to scale better in the presence of network topology dynamics and host mobility. In Section 8 we establish that LIS is a form of name-independent compact routing, discussed in Section 5 and using topology-unaware (flat) addressing. We show that contrary to common belief, the scaling properties of such routing can only be worse compared to the topology-aware, name-dependent case. We employ simulations to demonstrate that both the RT sizes and stretch induced by the best-performing name-independent schemes are significantly worse compared to their name-dependent counterparts. We conclude and pose open problems in Section 9 .

\section{INTERNET ROUTING TODAY}

There are two main related problems with the Internet's current routing architecture. First, it is not flexible, due primarily to the need to assign node addresses based on topological location. In order to achieve highly efficient address aggregation and relatively small routing tables, a CIDR network must have two characteristics: an essentially tree-like graph structure, and address assignment that follows this structure. Node mobility and end site multi-homing fundamentally break this model. But even recommended Internet traffic engineering techniques, e.g., load balancing, require de-aggregation of address blocks that cause the routing table to grow even if the network itself is not growing.

The second problem with the Internet's routing architecture is how the interdomain routing algorithm (path vector, for BGP) and its protocol implementation scale with the size of the network. Poor scaling of a routing algorithm expresses itself in terms of rapid, e.g., linear, rates of growth of the routing table (RT) size. Poor scaling of RT sizes exacerbates convergence problems (convergence time, churn, instabilities, etc.). Not only is the communication overhead of BGP known to be exponential [3, but the BGP RT size also appears to grow exponentially 4 .

The immediate causes of Internet RT growth have been extensively analyzed [1, 5, 6, 7, 8]; they reflect voluntary business relationships occurring at increasing density, traffic engineering techniques to improve robustness or reduce operational costs, and address allocation policies developed by IANA and the regional address registries - all resulting in various forms of de-aggregation, which is the primary cause of super-linear growth of the BGP RT size. Several studies, proposals, and even new routing architectures seek to mitigate these effects. Prominent efforts in this area include 9] [10, 11, 12, 13] and most recently [14, 15, 16]. However, these approaches do not rigorously provide non-trivial scaling guarantees, and many of them represent only short-term fixes since they address the symptoms rather than the root of the problem. More precisely, most of these approaches try to find shortest paths in the topology, leading to RT sizes that cannot scale better than linearly [17]. We are interested in algorithms that scale better than that. Ideally, we want to achieve logarithmic scaling ${ }^{2}$ that comes close to satisfy-

\footnotetext{
${ }^{2}$ To be more precise, we mean polylogarithmic scaling, but we abuse the terminology and do not differentiate between logarithmic and polylogarithmic growth in this paper.
}

ing our desire for "infinite scalability" in a future routing architecture [2].

As a representative example of the efforts mentioned above, we consider the idea of routing on autonomous system (AS) numbers, wherein AS numbers replace IP prefixes in the role of interdomain routing addresses. Using the coarser granularity of AS numbers is a natural idea, and is now a common part of many proposals [10, 12, 13, 14, 15. At first glance, routing on AS numbers looks like a solution to the RT size problem. Indeed, such an approach could immediately reduce the RT size by an order of magnitude, since there are on the order of $10^{5} \mathrm{IP}$ prefixes and $10^{4} \mathrm{AS}$ numbers in the global RT today. However, one can easily overlook that it is just a one-time constant-factor reduction, and not a change of the scaling behavior. As such, it is not a solution to the core problem, but rather a temporary relief measure. Indeed, if algorithms routing on AS numbers are still shortest-path routing algorithms, then they still cannot produce RT sizes that grow slower than linearly with the network size measured, in this case, by the total number of ASs in the Internet. To make things even worse, this number currently grows faster than the total number of IP prefixes [4, 7], and if the trend continues after a hypothetical adoption of routing on AS numbers, then the actual rate of growth of RT sizes can only be worse than today. Furthermore, routing on AS numbers requires some form of global directory to map IP addresses or prefixes to the AS numbers they are associated with.

So far we have argued that inflexibility of the current routing architecture precludes long-term scalability. An ideal routing architecture would allow for assigning node addresses independent of topology, and would employ routing algorithms that can truly scale. No proposal thus far has simultaneously solved both objectives; address aggregation remains the only generally accepted method for effectively limiting RT size growth, yet operational and business imperatives require intentional violation of this tenet. Something has to give.

In order to find sustainable solutions to the global Internet routing problem, we need to investigate routing scalability at its most fundamental level. For interdomain routing, the framing parameters are the performance guarantees of routing algorithms operating on graphs with topologies similar to the Internet AS-level topology, and which can support topology-independent addressing of nodes. In particular, we seek rigorous lower and upper bounds on the RT size used in supporting all routing features, i.e., mapping node names to their locations, and reaching those locations. Compact routing research establishes these bounds and constructs algorithms (or schemes) that try to meet them. One of the central themes in compact routing is the investigation of the inevitable trade-off between RT sizes and routing stretch.

\section{ROUTING STRETCH}

The stretch of a routing algorithm is defined as the worstcase path-length increase factor relative to shortest paths. More specifically, for every pair of nodes in all graphs in the set of graphs the algorithm can operate on, we find the ratio of the length of the route taken by the algorithm to the length of the shortest available path between the same pair of nodes. The maximum of this ratio among all node pairs in all the graphs in the set is the algorithm's stretch. 
The average stretch is the average of this ratio across all source-destination pairs in a given graph or set of graphs.

We emphasize that this notion of stretch has nothing in common with known forms of "path inflation" in contemporary Internet routing. Path inflation in today's Internet is not due to the stretch of the underlying routing algorithm, but rather due to intra- and inter-domain routing policies, and various incongruities across multiple levels (geographical, router, AS, etc.) of network topology abstraction 18 .

We are not aware of any widely-used routing protocol that produces other than stretch-1 (shortest) paths by default. Link-state (LS) in OSPF or ISIS, distance-vector (DV) in RIP or (E)IGRP, LS/DV hybrid in LVA [19, and pathvector (PV) in BGP, are all forms of trivial shortest-path routing (stretch-1). Consider two examples: BGP and OSPF. Non-trivial policy configurations generally prevent BGP from routing along the actual shortest path, but without policies the BGP route selection process would always select the shortest paths in the AS-level graph. Similarly, non-trivial area configurations prevent OSPF from routing along the shortest paths, but routing inside an area is always along the shortest path in the weighted router-level graph.

The above examples seem straightforward, but we identify them to eliminate any possible confusion between stretch of a routing algorithm and inflation of paths produced by a routing protocol subjected to policy or configuration constraints. Clarifying the difference allows us to formulate both necessary and sufficient conditions for the stretch of a routing algorithm proposed for use in the Internet.

The sufficient routing stretch requirement is that the routing algorithm underlying any realistic interdomain routing protocol must be of stretch-1. To understand the reasoning behind this requirement, consider a stretch $>1$ routing algorithm and apply it to a complete graph, which has all nodes directly connected to all other nodes so that all shortest paths are of length 1 . Since this algorithm does not guarantee finding all shortest paths, RTs at some nodes might lack entries corresponding to their own directly-connected neighbors. Put in different terms, in order to shrink RTs, the algorithm might have to force some nodes to delete information about some of their directly-attached interfaces.

Of course, the Internet interdomain topology is not a full mesh, and it might turn out that the same routing algorithm that fails to be stretch-1 on a full mesh does in fact find all shortest paths to each node's neighbors in realistic Internetlike topologies. We thus might also pursue the explicit task of finding a non-universal routing algorithm that is applicable only to Internet-like topologies. We do not require this algorithm be stretch-1, but it must be of stretch- 1 on paths of length 1 leading to nodes' neighbors. In other words, the necessary routing stretch requirement is that the routing algorithm underlying any realistic interdomain routing protocol must be of stretch-1 on paths of length 1 in realistic Internet-like topologies.

These requirements are purely practical: if a link exists between a pair of autonomous systems, it must be made available for carrying traffic. If our "sophisticated" routing algorithm removes routing information about such links from routing tables, then network operators will feel compelled to manually reinsert it, which counters the scalability objective of reducing the RT size and requires manual intervention which we would in general like to avoid. We will refer to this (undesirable) administrative re-adding of infor- mation about shortest paths to nodes' neighbors as manual reinsertion.

The absence of an immediate neighbor in a node's RT appears to contradict common sense, but one can verify that this situation can occur with the algorithms we discuss in Section 5. We have not had to deal with this effect in practice yet because the routing algorithms in operation today are all stretch-1. Stretch-1 algorithms require complete knowledge of the network topology (or at least of distances between nodes in it) and maintenance of an individualized optimal next hop for every possible destination in the network. This means $\Omega(n)$ entries are required in the RTs of every node in the worst case. In order to shrink RTs below this size, we must prepare to lose topological information, and consequently lose optimal next hops for some destinations. Balancing this fundamental and unavoidable trade-off between stretch of a routing algorithm and the sizes of RTs it produces is at the center of compact routing research today, but the first formalization of this trade-off was introduced in early work on hierarchical routing and addressing.

\section{HIERARCHICAL ROUTING, ADDRESS- ING, AND THEIR ALTERNATIVES}

In 1977 Kleinrock and Kamoun published their pioneering paper on hierarchical routing [20]. They showed how hierarchical clustering could be used with appropriate node addresses to produce highly scalable routing tables. This technique is the basis of the popular hierarchical routing approaches used today in practice, e.g., in the form of CIDR in the interdomain routing case, or usage of OSPF/ISIS areas in the intradomain routing case. The key idea is to group (or aggregate) nearby nodes into clusters, clusters into superclusters, and so on, in a bottom-up fashion. Determining these clusters can be accomplished through a hierarchical clustering algorithm, the performance of which is key to good routing performance.

Hierarchical aggregation and addressing offer substantial RT size reduction by abstracting out unnecessary topological details about remote portions of the network: nodes in one (super-)cluster need to keep only one RT entry for all nodes in another (super-)cluster. Almost all proposals for future Internet routing architectures trying to address the RT size problem are based, explicitly or implicitly, on this concept of hierarchical routing. In fact, no other concept behind RT size reduction has been systematically considered in the networking literature. Unfortunately, hierarchical routing is simply not a good candidate for interdomain routing, despite its current widespread use and reputation as realistically scalable, and we show why in the rest of this section.

In the same paper 20, Kleinrock and Kamoun were the first to analyze the stretch/RT size trade-off. They showed that the routing stretch produced by the hierarchical approach is satisfactory only for topologies with average shortest path hop-length (distance) $\bar{d}(n)$ that grows quickly with network size $n$, which means it performs well only for graphs where large distances between nodes prevail. In fact, they assumed that the average distance grows polynomially with the network size, $\bar{d}(n) \sim n^{\nu}$ with $\nu>0$. Such graphs have many remote nodes, i.e., nodes at long hop distances from each other, which allows for efficient aggregation of topology details without substantial path length increase. 


\subsection{Grids}

The simplest example of graphs with average distance $\bar{d}(n) \sim n^{\nu}$ are $\delta$-dimensional grids. The exponent $\nu$ is $1 / \delta$ in this case, and node aggregation works naturally: clusters are composed from nodes located at small Manhattan distances from each other, while super-clusters contain neighboring clusters, and so on.

We emphasize that aggregation is not the only technique to achieve logarithmic RT scaling for grids. We could alternatively embed a $\delta$-dimensional grid into a $\delta$-dimensional Euclidean space, and address nodes by their coordinates in the space. With such an embedding, nodes would not need to keep any RT information except for their own addresses, since knowing their own coordinates and the coordinates of the destination allows for shortest-path routing according to the Euclidean, i.e., Manhattan, distance. The RT size thus scales as the address size, i.e., logarithmically.

Logarithmic scaling extends from $\delta$-dimensional grids to graphs with doubling dimension $\delta$ [21]. The doubling dimension of a graph, or more generally, of a metric space, is the smallest $\delta$ such that every ball of radius $2 r$ can be covered by $2^{\delta}$ balls of radius $r$. Informally, such graphs are "perturbed versions" of $\delta$-dimensional grids. A key idea behind efficient addressing in such graphs allowing for logarithmic RT size growth is to introduce distance scales [22, 23] or maps of node coordinates. The address of a node becomes a collection of its coordinates, which are the distances from the node to carefully constructed subsets of nodes that form a "frame of reference." In other words, knowing the distances to these frame-of-reference subsets, we can uniquely identify a node's location in the graph. One can show then that in order to bring stretch arbitrarily close to 1 , we need only a logarithmic number of such subsets, which guarantees logarithmic RT sizes.

\subsection{Trees}

A strikingly similar situation occurs with routing on regular, i.e., $b$-ary, trees. They also allow for efficient aggregation: combine leaves of a single parent into a single cluster, while super-clusters contain clusters with a common parent of the parent, and so on.

Yet again we find that aggregation is not the only path toward logarithmic RT scaling on such trees. For example, we can use node addressing induced by a depth-first-search (DFS) node numbering. Given a DFS-based addressing convention, any node can infer its exact location on the tree from its address. In particular, a node knows what nodes are its children or descendants of which one of its children, and what nodes are not its descendants.

As with grids, logarithmic scaling extends from regular $b$-ary trees to a wider class of similar graphs - to arbitrary trees in this case [24, 25]. The main idea behind efficient addressing schemes is still to use DFS numbering, but we can no longer rely only on the node DFS ID to identify its location on a tree. It turns out however that it is possible to encode the path from the root of a tree to a node in such a way that this encoding preserves the logarithmic upper bound. If we now make the sequence of nodes lying on the path to the root a part of the node address, then one can check that routing along shortest paths becomes trivially possible.

\subsection{Grids and trees}

In both trees and grids, shortest-path routing is possible given only the addresses of the current and destination nodes. The RT size in either case scales logarithmically. This scaling performance can be extended beyond regular grids and trees to much wider classes of graphs, but not with hierarchical routing. The simplest example is routing on star graphs. These graphs are trees, but no hierarchical aggregation is possible on them. Logarithmic scaling in such cases is possible due only to specialized addressing techniques that leverage topological peculiarities of a graph's structure to succinctly encapsulate the location of a node into its address. Of course, these addressing techniques work trivially for regular grids and trees as well.

Recent progress in this area indicates that the efficiency of these types of addressing schemes is directly related to existence of low-distortion embeddings of finite metric spaces induced by shortest-path distances in grids and trees into normed spaces of low dimension 26. Low dimensions of host spaces guarantee small RT sizes, while low distortion of embeddings corresponds to small stretch. Grids embed in flat Euclidean spaces, but trees require spaces of negative curvature [27, 28].

\subsection{Scale-free graphs}

According to the best available data 29, the Internet topology is neither a tree nor a grid. Rather, it is scale-free. To avoid terminology disputes, by scale-free we simply mean networks with heavy-tail, e.g., power-law, node degree distributions and strong clustering, i.e., large numbers of triangular subgraphs 3 The latter characteristic implies that such graphs are not trees at all. The former, on the other hand, means that in theory, the average distance in them grows with the network size much more slowly than Kleinrock and Kamoun 20] assumed: at most $\bar{d}(n) \sim \log n$ 30. In practice, the average hop distance between autonomous systems in the Internet stays virtually constant or even decreases due to increasing inter-AS connectivity driven by economic or performance considerations 31, 7, 4. According to multiple data sources, the average AS-hop distance in the current Internet is between 3.1 and 3.7, with more than $80 \%$ of AS pairs being 2-4 hops away from each other 29. Topologies with small average distances are also called small worlds. There are essentially no remote nodes in small-world networks; all nodes are close to each other.

These characteristics are extremely bad news for hierarchical routing approaches, because the effectiveness of hierarchical network partitioning and aggregation depends either on the abundance of remote notes or on strong regularity of $b$-ary tree structure. None of these properties is present in scale-free graphs. In short, we are faced with an unsettling reality: one cannot hope to find a way to efficiently apply hierarchical, aggregation-based routing to Internet-like topologies.

These general arguments do not preclude the possibility of applying some generic hierarchical routing algorithm to the Internet. But they do imply that such an application cannot be efficient and we must prepare to see high stretch. Simple analytical estimates 32 show that applying hierarchical routing to an Internet AS-level topology incurs a $~ 15$-times

\footnotetext{
${ }^{3}$ There is an unfortunate terminological collision here: clustering means both grouping nodes into areas and presence of triangles in a graph.
} 
path length increase, which, although alarming enough by itself, would also lead to a substantial RT size surge caused by manual reinsertion.

If we accept our argument in Section 3 that any truly scalable Internet routing algorithm must have stretch as close as possible to 1 , we must also accept the fact that hierarchical routing will not meet our needs. Since all previous Internet interdomain routing proposals are heavily based on hierarchical routing, we recognize that while deploying one of them might offer short-term relief, none of them would genuinely scale to meet future demands.

\section{UNIVERSAL COMPACT ROUTING}

Compact routing research establishes fundamental limits for routing scalability, and constructs concrete routing algorithms (or schemes) that try to meet these limits. Formally, a routing scheme is said to be compact if it produces logarithmic address and header sizes, a sublinear RT size, and stretch bounded by a constant, as opposed to the growing stretch more typical of hierarchical routing 4 A routing scheme is universal if it works correctly and satisfies promised scaling bounds on all graphs. If it does so only on some specific graph classes, it is called specialized.

We can express fundamental limits of routing scalability using lower bounds on RT size versus stretch. For example, we can fix the maximum stretch and establish the minimum RT size for this stretch. Such a task usually involves constructing a concrete worst-case graph, enumerating RT size requirements of all possible routing schemes, and demonstrating that no routing scheme, applied to this worst-case graph, produces an RT smaller than the minimum 33. As soon as we have constructed a concrete routing scheme, we can establish its upper bounds, i.e., the maximum RT size and stretch it produces across all graphs. If a scheme's upper bound equals the theoretical lower bound, the scheme is said to be optimal.

The lower bounds for universal stretch-1 (shortest-path) routing are somewhat pessimistic. We first notice that we can construct RTs at each node by storing, for every destination node, the ID of the outgoing port on the shortest path to the destination. The number of destinations is $n-1$, the maximum number of ports a node can have (equal to maximum possible node degree) is also $n-1$, and thus a maximum $O(n \log n)$ bits of memory is required for an RT, i.e., $O(n \log n)$ is its upper bound. This construction is called trivial shortest-path routing, and all deployed LS-, DV-, or PV-based routing protocols implement it. However, Gavoille and Pérennès 17 showed that the lower bound of universal stretch- 1 routing is also $\Omega(n \log n)$, i.e., there is no shortest path routing scheme that for all nodes in all graphs, guarantees RTs smaller than the RTs of trivial shortest-path routing. In other words, shortest-path routing is incompressible. In order to decrease the maximum RT size, we therefore must allow maximum stretch to increase above 1 .

Gavoille and Genegler 33 showed that for any stretch strictly below 3 , the local space lower bound is nearly the same as for stretch-1 routing, $\Omega(n)$, meaning that no universal stretch $<3$ routing scheme can guarantee sublinear RT sizes: the minimum value of maximum stretch allowing sublinear RT sizes is 3. Thorup and Zwick [34] proved that any

\footnotetext{
${ }^{4}$ For example, one can verify that on an $n$-node full mesh, 20] produces stretch growing to infinity as $\Theta(\log n)$.
}

routing scheme with stretch strictly below 5 cannot guarantee space smaller than $\Omega\left(n^{1 / 2}\right)$. It is widely believed (although not yet proven) that stretch below $2 k-1$ imposes an RT size lower bound of $\Omega\left(n^{1 / k}\right)$ for all positive integers $k$.

Besides being universal or specialized, another important classification of routing schemes is whether they support name independence. Name-dependent schemes embed some topological information in node addresses (or labels) which thus cannot be arbitrary. We analyze a few classic examples of such addressing in Section 4 . Name-independent schemes work on graphs with arbitrary, e.g., flat, node labels. As discussed in Section 2 scalable name-independent schemes are highly desirable for future Internet routing. The distinction between these two cases is directly related to a popular discussion theme in the networking community, in which terms such as "node name" or "node identifier" essentially refer to the name-independent, topologically agnostic label, while terms "node address" or "node locator" usually imply a topologically informative node label, i.e., the namedependent label. Somewhat surprisingly, the lower and upper bounds for both name-dependent and name-independent universal routing are essentially the same.

\subsection{Name-dependent routing}

Kleinrock and Kamoun's hierarchical approach [20] was essentially the first name-dependent routing scheme. Besides problems discussed in Section 4 , their scheme provided no algorithm to construct a required partitioning for a given network. In subsequent works, first Kleinrock himself, then Perlman, and more recently Awerbuch, Peleg and others, invested much effort trying to find efficient network partitioning algorithms needed for correct operation of hierarchical routing.

It became evident that hierarchical routing approaches were intrinsically suboptimal when Cowen delivered her compact routing scheme [35. The scheme required no network partitioning, i.e., it was non-hierarchical, and it was fairly simple compared to numerous hierarchical routing algorithms accumulated by that time. It guaranteed the maximum stretch 3 and RT size of $\tilde{O}\left(n^{2 / 3}\right)$. Thorup and Zwick [25] (TZ) soon improved on Cowen's RT size upper bound, bringing it to $\tilde{O}\left(n^{1 / 2}\right)$ while maintaining stretch of 3 . The TZ scheme was thus the first nearly optimal universal stretch3 routing scheme because its RT size upper bound was nearly equal to the existing lower bound, up to logarithmic factors implied by " ' in the $\tilde{O}$ notation.

The TZ scheme is heavily based on the Cowen scheme. Both schemes first preprocess the graph and select a set of landmarks in it. In the Cowen scheme, this selection is based on results for dominating set construction, while the TZ scheme selects landmarks by means of randomized techniques. The landmark set sizes in the Cowen and TZ cases are $\tilde{O}\left(n^{2 / 3}\right)$ and $\tilde{O}\left(n^{1 / 2}\right)$, and that is essentially the only difference between the two schemes. The most non-trivial part in both the algorithms is to guarantee a proper balance between the sizes of landmark sets and clusters. Clusters are defined for every node $v$ in a graph, and $v$ 's cluster is the set of nodes that are closer to $v$ than to their corresponding closest landmarks. The TZ scheme, for example, guarantees that not only the landmark set size, but also the cluster size is upper-bounded by $\tilde{O}\left(n^{1 / 2}\right)$. The schemes then assign to all nodes $v$ the addresses, or labels, consisting of the following three parts: $v$ 's original identifier, the identifier of the 
landmark $L(v)$ closest to $v$, and the identifier of the interface at $L(v)$ that lies on the shortest path from $L(v)$ to $v$. The RT at $v$ consists of the next hops along shortest paths to all landmarks and all nodes in $v$ 's cluster. The RT size is thus $\tilde{O}\left(n^{1 / 2}\right)$. Node $v$ forwards a packet along the shortest path if the destination is either a landmark or a node in $v$ 's cluster. If $v$ is the closest landmark for the destination, $v$ forwards the packet along the shortest path using the interface identifier in the address in the packet header. Otherwise, $v$ forwards the packet to the landmark closest to the destination - its identifier is in the packet header and the next hop on the shortest-path route to it is in v's RT. It is a trivial exercise to check that the worst-case stretch experienced by such a routing scheme is 3 .

\subsection{Name-independent routing}

Abraham et al. 36] improved on previous results by Arias et al. 37. and delivered the first nearly optimal universal name-independent routing scheme, which had the same upper bounds as the name-dependent $\mathrm{TZ}$ scheme - maximum stretch of 3 and $\tilde{O}\left(n^{1 / 2}\right)$ RT sizes.

All name-independent schemes known to us consist of the following two parts:

- a name-dependent compact routing scheme operating underneath the name-independent one; and

- dictionary tables forming an efficiently-distributed database containing information necessary to translate nodes' name-independent addresses (flat identifiers) to name-dependent addresses (locators).

An important property of the dictionary tables is that they must be "everywhere dense" in the topology, so that any node $v$ can resolve all identifiers to locators by consulting dictionary tables of nodes located in $v$ 's neighborhood only. Obviously, node $v$ has to keep all routes to nodes in its neighborhood. Routing proceeds as follows: upon receiving a packet destined to identifier $X$, node $v$ forwards a packet to node $u$ in its neighborhood that according to the dictionary table construction rules (dependent on the routing scheme), contains an identifier-to-locator mapping for $X$. Upon reaching $u$, the packet learns the locator $x$ for $X$ and proceeds to $x$ as dictated by the underlying name-dependent scheme.

We emphasize that identifier-to-locator mapping is an integral part of the routing process, and all performance bounds apply to the combined process of mapping and forwarding. Specifically, name-independent RTs include both the RTs of underlying name-dependent schemes and the dictionary tables, whereas total stretch accounts for travel to a nameresolution node and then from it to the destination along a name-dependent, potentially stretched route. This is in contrast to the separation of DNS name resolution and IP routing in the Internet, where DNS is essentially an application running on top of IP and requiring routing to work in the first place. It is therefore remarkable that there exist both name-dependent and name-independent universal stretch-3 compact routing schemes with the same $\tilde{O}\left(n^{1 / 2}\right)$ RT size upper bound.

\section{COMPACT ROUTING ON SCALE-FREE GRAPHS}

One might be alarmed by the previous section, as it seems to conflict with Section 3 where we show that because of practical reasons, stretch must be close to 1 . The combination of shortest-path routing incompressibility and the impossibility of stretch $<3$ routing with sublinear RT sizes seem to imply that scalable interdomain routing is impossible. Recall, however, that the stretch values mentioned in Section 5 refer to the maximum stretch across all paths in all graphs, meaning that there exists some worst-case graph on which this maximum is achieved. More importantly, all schemes mentioned in Section 5]are universal. Since they can work on all graphs, the worst-case graph may not be, and usually is not, "Internet-like." We can consequently expect the average stretch of these schemes on scale-free graphs be lower than 3. But how close is it to 1 ?

At first glance, it seems unlikely that it could be too close to 1 , since we saw in Section 4.4 that the stretch of hierarchical routing is high on Internet-like graphs, and there is no apparent reason to believe that compact routing would be drastically better. In 32 however, the authors showed that the average performance of the first optimal stretch-3 scheme (TZ) 25] on scale-free topologies is much better than its worst case. While TZ's upper bounds are around 2200 for RT size and 3 for stretch, the average RT size was found to be around 50 entries for the whole AS-level Internet, and the average stretch was only 1.1! These numbers are striking evidence that, on the same topology and under the same assumptions, compact routing has dramatically superior scaling characteristics compared to hierarchical routing 20], with its stretch of 15 and RTs of unbounded size, and compared to simple routing on AS numbers (e.g., 15]), with RT sizes of the order of $10^{4}$.

The observation in 32] that scale-free graphs yield essentially the best possible performance of the TZ scheme compared to all other graphs was the first indication that scalefree graphs are optimally structured for high-performance routing. This indication inspired several research groups to work on construction of specialized routing schemes 38 , 39] designed to utilize structural peculiarities of scale-free graphs in order to improve routing performance guarantees. The schemes in 38, 39] achieve logarithmic RT scaling and infinitesimally small stretch on scale-free graphs. Both schemes are based on the observation that although scale-free graphs are not trees, trees cover scale-free graphs without incurring much stretch.

As a representative example, the scheme by Brady and Cowen 38] ("BC scheme") grows one shortest-path tree rooted at the highest-degree node and a small fixed number of additional trees to cover edges in the fringe of a power-law graph. Routing is then confined to this tree collection according to the schemes discussed in Section 4.2 Since their RT sizes scale logarithmically, the BC scheme achieves logarithmic scaling. Specifically, it guarantees a maximum RT size of $O\left(\log ^{2} n\right)$. The $O\left(\log ^{2} n\right)$ scaling means that even if the graph size is $2^{128}$, i.e., if all IPv6-capable devices independently participate in global routing, then the maximum routing table size in such an Internet would still contain not more than $128^{2} \sim 16,000$ entries! The corresponding limit for $\mathrm{IPv} 4$ is $\sim 1,000$.

\footnotetext{
${ }^{5}$ See [33] for an example of these worst-case graphs.
} 
We thus see that even though hierarchical routing approaches based on address aggregation cannot work efficiently on Internet-like topologies (Section 4), more modern routing algorithms achieve essentially "infinite" scalability on scale-free graphs, if they are static. Can there exist any routing schemes that scale as well on dynamic networks?

\section{DYNAMIC ROUTING ON SCALE-FREE GRAPHS}

One of the most limiting assumptions behind the routing algorithms we have considered thus far is that they assume that the network is effectively static. More precisely, it is not that the network is actually static per se, but that the routing algorithms require a full view of the graph representing the network topology at any given instant in time. Any topology change yields a new graph, on which the algorithm might have to perform its calculations from scratch. This behavior is characteristic of currently-deployed Internet routing algorithms as well.

The most basic parameter measuring the dynamic performance of routing algorithms is the communication cost - the number of control messages needed to converge after a topology change. Communication costs are as important as RT size or stretch. They are associated with nodes requiring properly updated information about the network topology in order to route. Deployed routing protocols, for example, require all nodes to have a coherent full view of the network topology or of the distances in it. Indeed, link-state algorithms, typically used for intra-domain routing, require each node to have a complete view of all links in the network, and each node performs a shortest-path computation on essentially a static graph. Distance- or path-vector algorithms need each node to know distances or paths to all other nodes in the network.

In order to achieve such coherent full views of the network topology, timely update messages, a.k.a. routing updates, are employed. Updates requiring recalculation of routing tables can lead to delay, instabilities, churn, and other complications. For example, there are BGP oscillation scenarios with infinite communication costs 40, 41, and even during more typical BGP behavior, they are exponential [3]. High communication costs and resulting long convergence times are both a critical problem and an absolutely inevitable implication of growth of the current routing architecture [1.

Using fairly general arguments, Afek et al. [42] showed that the communication cost cannot scale better than $\Omega(n)$. Their results applied to universal schemes, so we could still hope that schemes specialized for scale-free graphs would behave better, as the $\Omega(n)$ bound could be attained only on some marginal worst-case graphs. After seventeen years of little progress on this problem, Korman and Peleg have recently tried to improve this pessimistic lower bound in 43 . Unfortunately for Internet routing, their results are even more pessimistic than Afek's since we use them, combined with simple analytic estimates, to demonstrate in the Appendix that the communication cost lower bound for scalefree graphs is $\tilde{\Omega}(n)$. This finding means that as far as communication costs are concerned, Internet-like graphs belong to the class of worst-case graphs, across all possible network topologies!

\section{LOCATOR-IDENTIFIER SPLIT (LIS)}

Despite all the findings mentioned above, a popular proposed approach to Internet routing scalability and flexibility is to separate the identifier and locator of a node in the network. In an architecture where one label identifies a node and a different label indicates its location, topology changes will only change the locators, which are assumed to follow topology and allow for aggressive aggregation. Other functions (access control lists, application and transport layer identification of endpoints) can use the identifiers. This would support significantly enhanced flexibility of the Internet routing system, but does it really address the scalability concerns?

In light of the discussion in Sections 4.4, 5.2 and 7 , we must admit that LIS cannot really improve routing scalability, for at least two reasons:

- Since LIS approaches are based, explicitly or implicitly, on aggressive aggregation of locators, they cannot improve scaling of RT sizes because such aggregation is impossible on scale-free topologies;

- Even if LIS approaches did not use aggressive aggregation, they still could not improve scaling, because in addition to maintaining and updating RTs of locators as before, the networks must also maintain and update a (distributed) database of identifier-to-locator mappings.

To ground these claims more firmly, we first observe that name-independent routing from Section 5.2 naturally implements LIS. In fact, the only conceptual difference between name-independent routing and LIS is that the former is more flexible than the latter, since it does not assume that locators, i.e., name-dependent addresses, are amenable to aggressive aggregation. It is obvious, however, that the average scaling characteristics of name-independent schemes cannot be better than name-dependent ones, since the name independent schemes are essentially name dependent schemes plus something else, i.e., plus dictionary tables and identifierto-locator name-resolution, which incurs both RT size increase and stretch, cf. Section 5.2

To experimentally confirm this reasoning, we implemented the best-performing name-dependent and name-independent schemes. Specifically, among name-dependent schemes, we chose the universal optimal stretch-3 TZ scheme [25] and the $\mathrm{BC}$ scheme 38] designed for scale-free graphs. We also tested the TZ/BC hybrid scheme, which simply selects the lower-stretch path by running both schemes in parallel. For the name-independent case, we tested the optimal universal Abraham scheme 36. We then applied all schemes to AS-level Internet topologies measured by skitter [44] and DIMES [45, and calculated the resulting average RT size and stretch. Table1 1 and Figure 1 show the outcome of these experiments, revealing that all characteristics of the nameindependent routing are significantly worse than their namedependent counterparts. By no means do these observations conflict with our statements in Section 5 that the worst-case scaling of name-dependent and name-independent universal routing schemes is the same. They do show, however, that name-independent scaling on Internet topologies is much worse on average.

Furthermore, our findings are consistent with the scaling properties of name-independent routing on trees. We 
Table 1: The average stretch and routing table sizes (in entries and bits) that the best performing namedependent (BC, TZ, and their hybrid TZ/BC) and name-independent (Abraham) schemes produce on the skitter and DIMES topologies.

\begin{tabular}{|l|rr|rr|}
\hline Scheme & \multicolumn{2}{|c|}{ skitter (9204 nodes) } & DIMES (13931 nodes) \\
\hline TZ & 62 entries (1629 bits), 1.08 stretch & 69 entries (1887 bits), & 1.13 stretch \\
BC & 22 entries (1025 bits), & 1.06 stretch & 22 entries (1103 bits), & 1.03 stretch \\
TZ/BC hybrid & 84 entries (2654 bits), & 1.02 stretch & 91 entries (2990 bits), & 1.01 stretch \\
Abraham & 3251 entries (240427 bits), & 1.35 stretch & 4145 entries (326781 bits), & 1.45 stretch \\
\hline
\end{tabular}

mention trees here because tree routing from Section 4.2 forms the core of routing schemes specialized for Internet topologies in Section 6. Unfortunately, as found in [46, 47, name-independent routing on trees cannot scale better than on general graphs. In other words, trees are the worst-case graphs for universal name-independent routing 6 The reason for that is simple: trees cannot guarantee that nodes' neighborhoods, i.e., balls of small radii that store dictionary tables, comprise only small portions of the graph. Examples of graphs that satisfy this property are grids or their perturbations from Section 4.1 i.e., graphs of low doubling dimension. The average distance in these graphs grows quickly with the graph size, the relative size of nodes' neighborhoods can thus be made small, and as a consequence, the RT sizes of name-independent routing scale logarithmically on such graphs [21]. We summarize these scaling characteristics in Table 2

\section{CONCLUSION}

Compact routing is a research area that aims at solving the following two classes of problems: 1) identify the fundamental scaling limits (lower bounds) of routing on graphs; and 2) construct routing algorithms that meet those limits, i.e., whose upper bounds are equal to the corresponding lower bounds. In other words, compact routing reveals the most fundamental properties of routing scalability, refined from layers of complexity associated with routing in practice.

As such, compact routing algorithms offer remarkably better scaling than we observe in the Internet routing today. In particular, existing interdomain routing exhibits exponentially growing routing table sizes, while those of compact routing algorithms designed for Internet-like topologies scale logarithmically.

Logarithmic scaling is essentially the only scaling behavior that satisfies the requirement for a future routing architecture to "scale indefinitely" 2]. Unfortunately, only static and name-dependent, i.e., topology-aware, routing exhibit logarithmic scaling on observed Internet topologies.

If name-independence is a requirement, i.e., if we need to route on topology-unaware flat identifiers, then routing tables cannot scale better than polynomially on Internetlike topologies. The lower bound is $\Omega(n)$ for shortest-path routing, $\Omega(\sqrt{n})$ for stretch-3 routing, and more generally, $\Omega\left(n^{1 / k}\right)$ for routing with stretch of $2 k-1, k=1,2, \ldots$

Worse than that, the communication cost, i.e., the number of routing control messages per topology change, cannot grow slower than linearly on Internet-like topologies. Of course, this scaling is still much better than the exponential

\footnotetext{
${ }^{6}$ More specifically, Abraham et al. 47 explicitly use stars
} to prove the name-independent lower bounds. communication costs of deployed routing protocols, but linear scaling can definitely not be considered as satisfying the requirement of "infinite scalability."

We are at an apparent impasse. Routing schemes specialized for static Internet-like graphs scale essentially indefinitely, but in adding the associated updates required to handle dynamic graphs, we can no longer guarantee the required scaling behavior. We must face the reality that scalable routing which uses topology update messages to dynamically react to topology changes is not possible in principle, which explains, in part, the lack of significant progress in search of truly scalable routing. We thus conclude that in order to find approaches that would lead us to required routing scalability, we need some radically new ideas that would allow us to construct convergence-free, "updateless" routing requiring no full view of network topologies. It is widely known and popularized that having no full view of social network topologies, humans can still efficiently route messages through them [48. Whether we can devise routing protocols that would do the same for the Internet is an open question.

\section{ACKNOWLEDGEMENTS}

This work is supported in part by NSF CNS-0434996.

\section{REFERENCES}

[1] D. Meyer, L. Zhang, and K. Fall (Eds.). Report from the IAB workshop on routing and addressing. IRTF, Internet Draft, 2007. http://www.ietf .org/internet-drafts/draft-iab-raws-report-

[2] A. Doria, E. Davies, and F. Kastenholz (Edts.). Requirements for inter-domain routing. IRTF, Internet Draft, 2006.

[3] C. Labovitz, A. Ahuja, A. Bose, and F. Jahanian. Delayed Internet routing convergence. Transactions on Networking, 9(3), 2001.

[4] BGP Table Data, http://bgp.potaroo.net/.

[5] G. Huston. Scaling inter-domain routing - a view forward. The Internet Protocol Journal, 4(4), 2001.

[6] G. Huston. Commentary on inter-domain routing in the Internet. IETF, RFC 3221, 2001.

[7] A. Broido, E. Nemeth, and kc claffy. Internet expansion, refinement, and churn. European Transactions on Telecommunications, 13(1):33-51, 2002.

[8] H. Narayan, R. Govindan, and G. Varghese. The impact of address allocation and routing on the structure and implementation of routing tables. In SIGCOMM, 2003.

[9] Beyond BGP. http://www. beyondbgp.net/ 

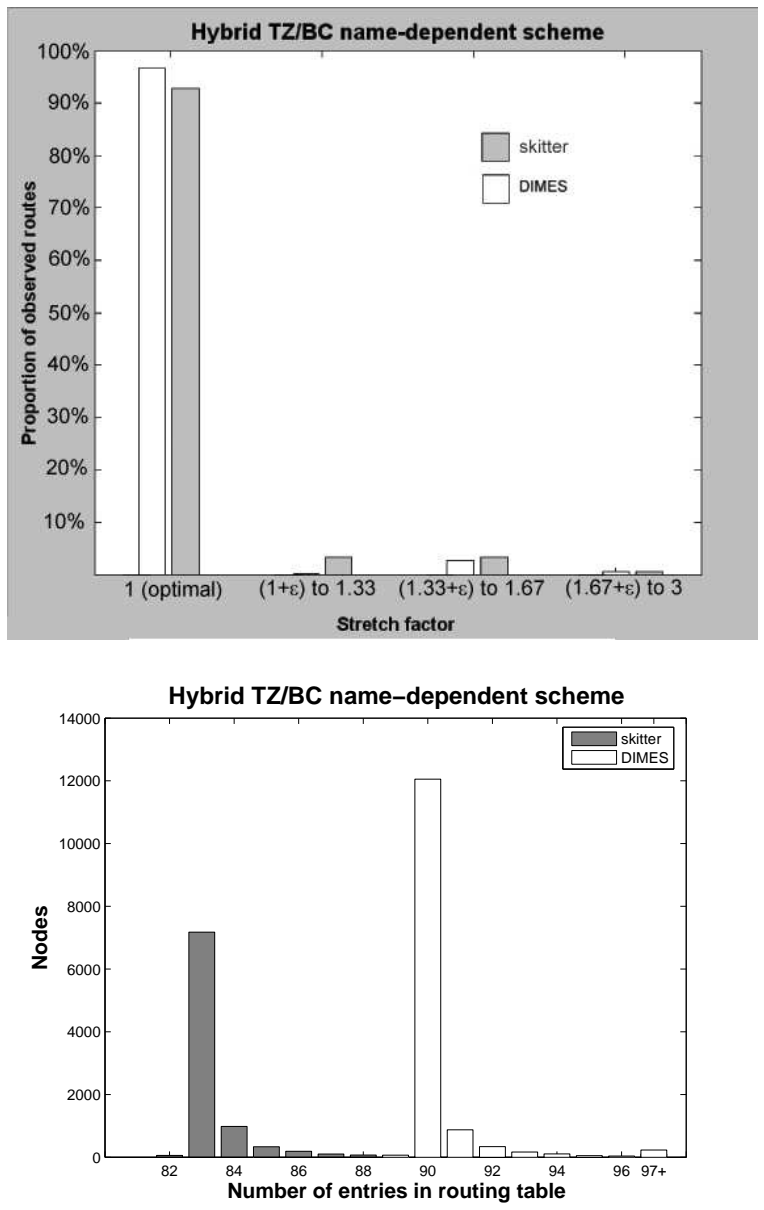
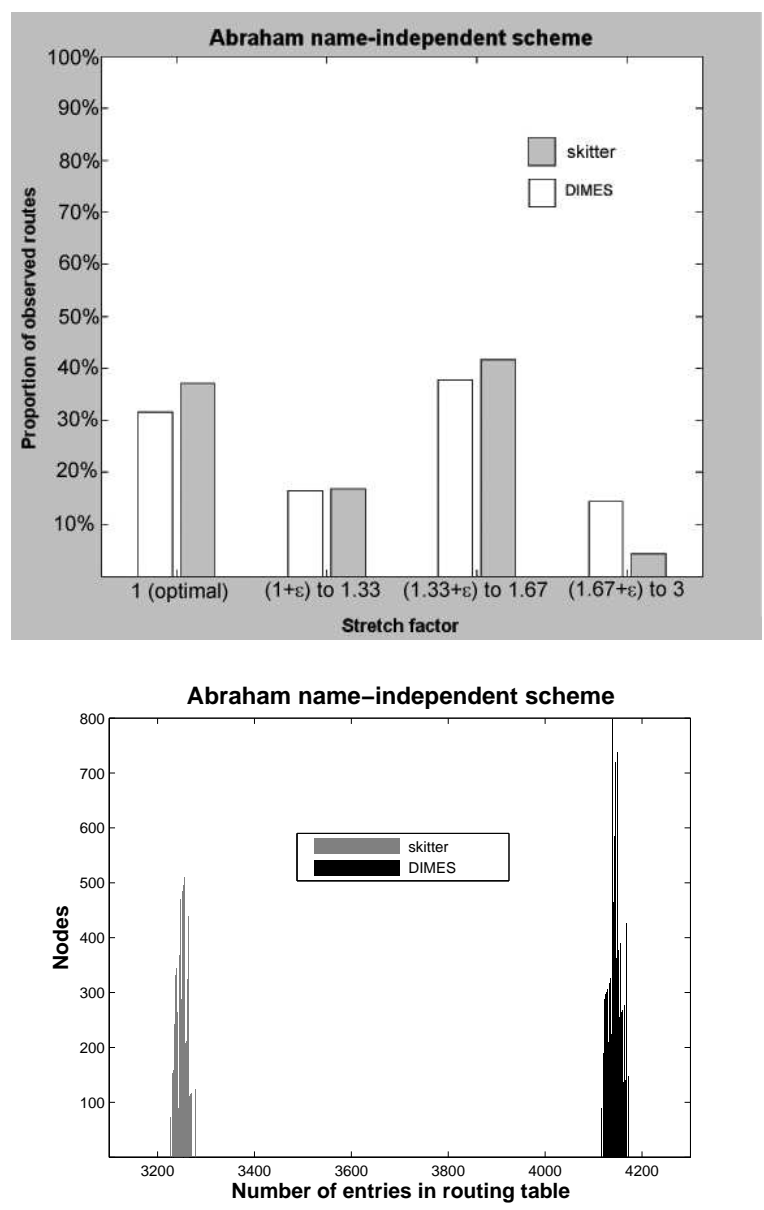

Figure 1: The distributions of stretch (top row) and routing table size (bottom row) that the hybrid TZ/BC (left column) and Abraham (right column) schemes produce on the skitter and DIMES topologies.

[10] P. Verkaik, A. Broido, kc claffy, R. Gao, Y. Hyun, and R. van der Pol. Beyond CIDR aggregation. Technical Report TR-2004-1, CAIDA, 2004.

[11] Internet Engineering Task Force (IETF). Site multihoming in IPv6. Working Group.

[12] F. Kastenholz. ISLAY: A new routing and addressing architecture. IRTF, Internet Draft, 2002.

[13] I. Castineyra, N. Chiappa, and M. Steenstrup. The nimrod routing architecture. IETF, RFC 1992, 1996.

[14] R. Gummadi, R. Govindan, N. Kothari, B. Karp, Y.-J. Kim, and S. Shenker. Reduced state routing in the Internet. In HotNets, 2004.

[15] L. Subramanian, M. Caesar, C. T. Ee, M. Handley, M. Mao, S. Shenker, and I. Stoica. HLP: A next generation inter-domain routing protocol. In SIGCOMM, 2005.

[16] M. Caesar, T. Condie, J. Kannan, K. Lakshminarayanan, I. Stoica, and S. Shenker. ROFL: Routing on flat labels. In SIGCOMM, 2006.

[17] C. Gavoille and S. Pérennès. Memory requirement for routing in distributed networks. In $P O D C, 1996$.

[18] N. Spring, R. Mahajan, and T. Anderson. Quantifying the causes of path inflation. In SIGCOMM, 2003.
[19] J. Behrens and J. J. Garcia-Luna-Aceves. Distributed, scalable routing based on link-state vectors. In SIGCOMM, 1994.

[20] L. Kleinrock and F. Kamoun. Hierarchical routing for large networks: Performance evaluation and optimization. Computer Networks, 1:155-174, 1977.

[21] I. Abraham, C. Gavoille, A. Goldberg, and D. Malkhi. Routing in networks with low doubling dimension. In ICDCS, 2006.

[22] P. Indyk and J. Matoušek. Handbook of Discrete and Computational Geometry, chapter 8, Low-Distortion Embeddings of Finite Metric Spaces. Chapman \& Hall/CRC, Boca Raton, 2004.

[23] I. Abraham, Y. Bartal, T.-H. H. Chan, K. Dhamdhere, A. Gupta, J. Kleinberg, O. Neiman, and A. Slivkins. Metric embeddings with relaxed guarantees. In FOCS, 2005.

[24] P. Fraigniaud and C. Gavoille. Routing in trees. In ICALP, 2001.

[25] M. Thorup and U. Zwick. Compact routing schemes. In $S P A A, 2001$.

[26] J. Matoušek. Lectures on Discrete Geometry, chapter 15, Embedding Finite Metric Spaces into Normed Spaces. Springer, New York, 2002. 
Table 2: Routing table (RT) size scaling for name-dependent and name-independent routing on different graph classes. Both "Logarithmic" and "Polynomial" tags in this table have specific meanings. "Logarithmic" means that RT sizes scale as $\Omega(\log n)$ even for shortest-path (stretch-1) routing. "Polynomial" means $\Omega(n)$ for stretch-1 routing, $\Omega(\sqrt{n})$ for stretch-3 routing, and more generally, $\Omega\left(n^{1 / k}\right)$ for routing with stretch of $2 k-1$, $k=1,2, \ldots$.

\begin{tabular}{|l|l|l|}
\hline Graph class & Name-dependent routing & Name-independent routing \\
\hline General graphs & Polynomial & Polynomial \\
Trees & Logarithmic & Polynomial \\
Grids & Logarithmic & Logarithmic \\
\hline
\end{tabular}

[27] R. Krauthgamer and J. R. Lee. Algorithms on negatively curved spaces. In FOCS, 2006.

[28] R. Kleinberg. Geographic routing using hyperbolic space. In INFOCOM, 2007.

[29] P. Mahadevan, D. Krioukov, M. Fomenkov, B. Huffaker, X. Dimitropoulos, kc claffy, and A. Vahdat. The Internet AS-level topology: Three data sources and one definitive metric. Computer Communication Review, 36(1), 2006.

[30] F. Chung and L. Lu. The average distance in a random graph with given expected degrees. Internet Mathematics, 1(1):91-114, 2003.

[31] J. Leskovec, J. Kleinberg, and C. Faloutsos. Graphs over time: Densification laws, shrinking diameters and possible explanations. In $K D D, 2005$.

[32] D. Krioukov, K. Fall, and X. Yang. Compact routing on Internet-like graphs. In INFOCOM, 2004.

[33] C. Gavoille and M. Genegler. Space-efficiency for routing schemes of stretch factor three. Journal of Parallel and Distributed Computing, 61(5):679-687, 2001.

[34] M. Thorup and U. Zwick. Approximate distance oracles. In $S T O C, 2001$.

[35] L. Cowen. Compact routing with minimum stretch. Journal of Algorithms, 38(1):170-183, 2001.

[36] I. Abraham, C. Gavoille, D. Malkhi, N. Nisan, and M. Thorup. Compact name-independent routing with minimum stretch. In SPAA, 2004.

[37] M. Arias, L. Cowen, K. A. Laing, R. Rajaraman, and O. Taka. Compact routing with name independence. In $S P A A, 2003$.

[38] A. Brady and L. Cowen. Compact routing on power-law graphs with additive stretch. In ALENEX, 2006.

[39] S. Carmi, R. Cohen, and D. Dolev. Searching complex networks efficiently with minimal information. Europhysics Letters, 74:1102-1108, 2006.

[40] T. Griffin and G. Wilfong. An analysis of BGP convergence properties. In SIGCOMM, 1999.

[41] T. Griffin and G. Wilfong. Analysis of the MED oscillation problem in BGP. In ICNP, 2002.

[42] Y. Afek, E. Gafni, and M. Ricklin. Upper and lower bounds for routing schemes in dynamic networks. In FOCS, 1989.

[43] A. Korman and D. Peleg. Dynamic routing schemes for general graphs. In ICALP, 2006.

[44] CAIDA. Macroscopic topology AS adjacencies. http://www.caida.org/tools/measurement/skitter/as_adjacencies.xml

[45] The DIMES project. http://www.netdimes.org/.
[46] K. A. Laing and R. Rajaraman. A space lower bound for name-independent compact routing in trees. In SPAA, 2005.

[47] I. Abraham, C. Gavoille, and D. Malkhi. On space-stretch trade-offs: Lower bounds. In $S P A A$, 2006.

[48] S. Milgram. The small world problem. Psychology Today, 1:61-67, 1967.

[49] S. N. Dorogovtsev, J. F. F. Mendes, and A. N. Samukhin. Metric structure of random networks. Nuclear Physics B, 653(3):307-422, 2003.

\section{APPENDIX}

We demonstrate that the lower bound for communication costs in random scale-free graphs of size $n$ is $\tilde{\Omega}(n)$. The average distance $\bar{d}$ in such graphs grows logarithmically with $n$, $\bar{d} \sim \log n$ 30, 49, while the width (standard deviation) of the distance distribution approaches zero in the large graph limit 49]. The latter property implies that the distance distribution $d_{x}(r)$ for every node $x$, i.e., the number of nodes located at distance $r$ from $x$ divided by $n$, approaches a delta-function as $n \rightarrow \infty$ :

$$
d_{x}(r) \underset{n \rightarrow \infty}{\longrightarrow} \begin{cases}1 & \text { if } r=\bar{d} \sim \log n, \\ 0 & \text { otherwise. }\end{cases}
$$

Korman and Peleg [43] define the local density as

$$
D=\max _{x, r} \frac{\left|B_{x}(r)\right|}{2 r},
$$

where $B_{x}(r)$ is the ball of radius $r$ around node $x$, i.e., the set of nodes located within distance at most $r$ from $x$, and $\left|B_{x}(r)\right|$ is the size of this set. The maximum is taken over all nodes $x$ in the graph and all radii $r$. Observing that $\left|B_{x}(r)\right|=n \int_{0}^{r} d_{x}(\rho) d \rho$ and using eq. (1), we immediately obtain that $\left|B_{x}(r)\right|$ in large scale-free graphs is given by the Heaviside function:

$$
\left|B_{x}(r)\right| \underset{n \rightarrow \infty}{\longrightarrow} \begin{cases}n & \text { if } r \geqslant \bar{d} \sim \log n \\ 0 & \text { otherwise. }\end{cases}
$$

Substitution of this expression into eq. (2) yields

$$
D \underset{n \rightarrow \infty}{\longrightarrow} \frac{n}{\log n} \text {. }
$$

The last expression and the demonstration in 43] that the communication cost lower bound for graphs with local density $D$ is $\Omega(D)$ complete the proof. 